\title{
Filtering number states of the vibrational motion of an ion
}

\author{
H. Moya-Cessa* and P. Tombesi \\ Via Madonna delle Carceri, Dipartimento di Matematica e Fisica, Università di Camerino and \\ INFM unità Camerino, I-62032 Camerino (MC), Italy
}

(September 14, 2018)

\begin{abstract}
We propose a scheme to generate number states, and specific superpositions of them, of the vibrational motion of a trapped ion via laser excitation of two vibronic transitions. Without detrimental effects on the internal state preparation, the ion can be initially in thermal motion and undergo spontaneous emission. In particular, robust to noise qubits $(\alpha|0\rangle+\beta|1\rangle$ states) can be generated with arbitrary amplitudes.

42.50.Vk, 03.65.-w, 32.80.Pj
\end{abstract}

Typeset using REVTEX

*On leave from: INAOE, Coordinación de Optica, Apdo. Postal 51 y 216, 72000 Puebla, Pue., Mexico 
Recently major efforts have been directed towards the generation of non-classical states of light [1,2] and vibrational motion of ions [3]. In particular, number states and specific superpositions of them are of importance for a number of different applications ranging from spectroscopy to fundamental tests of quantum mechanics. Methods to engineer quantum states of light have already been proposed which are mainly based on conditional measurements [1.5]. There have been proposed methods to restore [6] and protect [7] states and also to engineer not a state itself but the Hamiltonian [8]. Here we propose to generate number states and given superpositions of them, with controllable spacing, by bicromatic excitation of an ion which can be realized with present available technology. Our scheme is based on proposals by de Matos Filho and Vogel where they show that Schrödinger cats [3] and nonlinear coherent states [4] emerge from the long time dynamics, when the ion decouples from the laser field and remains in a pure state. In this contribution, by using the same scheme as in [3, 4] we exploit the fact that, by manipulating the Lamb-Dicke parameters (LDPs) of both fields, under certain conditions we can generate superpositions of spaced number states below a given definite number, above it, or generate a single number state, therefore "filtering" (choosing) number states. In particular, robust to noise qubits [9] $(\alpha|0\rangle+\beta|1\rangle$ states) can be generated with arbitrary amplitudes.

We consider the Hamiltonian of a single ion trapped in a harmonic potential in interaction with laser light in the rotating wave approximation, which reads

$$
\hat{H}=\hbar \nu \hat{a}^{\dagger} \hat{a}+\hbar \omega_{21} \hat{A}_{22}+\hbar\left[\lambda E^{(-)}(\hat{x}, t) \hat{A}_{12}+H . c .\right]
$$

where $\hat{a}$ and $\hat{A}_{a b}$ are the annihilation operator of a quantum of the ionic vibrational motion and the electronic (two-level) flip operator for the $|b\rangle \rightarrow|a\rangle$ transition of frequency $\omega_{21}$, respectively. $\nu$ is the trap frequency, $\lambda$ the electronic coupling matrix element, and $E^{(-)}(\hat{x}, t)$ the negative part of the classical electric field of the driving field.

We assume the ion driven by two laser fields, the first tuned to the $j$ th lower sideband and the second tuned to the $m$ th lower sideband, therefore we may write $E^{(-)}(\hat{x}, t)$ as

$$
E^{(-)}(\hat{x}, t)=E_{j} e^{-i\left(k_{j} \hat{x}-\omega_{21}+j \nu\right) t}+E_{m} e^{-i\left(k_{m} \hat{x}-\omega_{21}+m \nu\right) t}
$$


where, if $m=0$ it would correspond to the driving field being on resonance with the electronic transition, and $\hat{x}$ may be written as

$$
k_{s} \hat{x}=\eta_{s}\left(\hat{a}+\hat{a}^{\dagger}\right)
$$

where $k_{s}$ are the wave vectors of the driving fields and

$$
\eta_{s}=2 \pi \frac{\sqrt{\left\langle 0\left|\Delta \hat{x}^{2}\right| 0\right\rangle}}{\lambda_{s}}
$$

are the LDPs with $s=j, m$.

It has been noted recently, that approximations based on this parameter can lead to divergencies in the mean motional excitation [10]. A way of linearizing the Hamiltonian (1) which accounts for all orders of the Lamb-Dicke parameter and any sideband regime has been proposed in [11].

In the resolved sideband limit, the vibrational frequency $\nu$ is much larger than other characteristic frequencies and the interaction of the ion with the two lasers can be treated separately, using a nonlinear Hamiltonian [3]. The Hamiltonian (1) in the interaction picture can then be written as

$$
\hat{H}_{I}=\hbar \hat{A}_{21}\left[\Omega_{j} e^{-\eta_{j}^{2} / 2} \frac{\hat{n} !}{(\hat{n}+j) !} L_{\hat{n}}^{(j)}\left(\eta_{j}^{2}\right) \hat{a}^{j}+\Omega_{m} e^{-\eta_{m}^{2} / 2} \frac{\hat{n} !}{(\hat{n}+m) !} L_{\hat{n}}^{(m)}\left(\eta_{m}^{2}\right) \hat{a}^{m}\right]+\text { H.c. }
$$

where $L_{\hat{n}}^{(k)}\left(\eta_{k}^{2}\right)$ are the operator-valued associated Laguerre polynomials and the $\Omega$ 's are the Rabi frequencies and $\hat{n}=\hat{a}^{\dagger} \hat{a}$. The master equation which describes this system can be written as

$$
\frac{\partial \hat{\rho}}{\partial t}=-\frac{i}{\hbar}\left[\hat{H}_{I}, \hat{\rho}\right]+\frac{\Gamma}{2}\left(2 \hat{A}_{12} \hat{\tilde{\rho}} \hat{A}_{21}-\hat{A}_{22} \hat{\rho}-\hat{\rho} \hat{A}_{22}\right)
$$

where the last term describes spontaneous emission with energy relaxation rate $\Gamma$, and

$$
\hat{\tilde{\rho}}=\frac{1}{2} \int_{-1}^{1} d s W(s) e^{i s \eta_{E} \hat{x}} \hat{\rho} e^{-i s \eta_{E} \hat{x}}
$$

accounts for changes of the vibrational energy because of spontaneus emission. Here $\eta_{E}$ is the LDP corresponding to the field (2) and $W(s)$ is the angular distribution of spontaneus emission [3, [4]. 
The steady-state solution to Eq. (6) is obtained by setting $\partial \hat{\rho} / \partial t=0$ and may be written as [3, [1]

$$
\hat{\rho}_{s}=|1\rangle\left|\psi_{s}\right\rangle\left\langle\psi_{s}\right|\langle 1|
$$

where $|1\rangle$ is the electronic ground state and $\left|\psi_{s}\right\rangle$ is the vibrational steady-state of the ion, given by

$$
\left(\Omega_{j} e^{-\eta_{j}^{2} / 2} \frac{\hat{n} !}{(\hat{n}+j) !} L_{\hat{n}}^{(j)}\left(\eta_{j}^{2}\right) \hat{a}^{j}+\Omega_{m} e^{-\eta_{k}^{2} / 2} \frac{\hat{n} !}{(\hat{n}+m) !} L_{\hat{n}}^{(m)}\left(\eta_{m}^{2}\right) \hat{a}^{m}\right)\left|\psi_{s}\right\rangle=0 .
$$

For simplicity, we will concentrate in the $j=1$ and $m=0$ case (single number state spacing) for which Eq. (9) is written as

$$
\left(\Omega_{1} e^{-\eta_{1}^{2} / 2} \frac{L_{\hat{n}}^{(1)}\left(\eta_{1}^{2}\right)}{\hat{n}+1} \hat{a}+\Omega_{0} e^{-\eta_{0}^{2} / 2} L_{\hat{n}}\left(\eta_{0}^{2}\right)\right)\left|\psi_{s}\right\rangle=0 .
$$

Note that $\hat{H}_{I}|1\rangle\left|\psi_{s}\right\rangle=0$ so that ion and laser have stopped to interact, which occurs when the ion stops to fluoresce. For the $j=1$ and $k=0$ case, and assuming $L_{k}^{(1)}\left(\eta_{1}^{2}\right) \neq 0$ and $L_{k}\left(\eta_{0}^{2}\right) \neq 0$ for all $k$, one generates nonlinear coherent states [4]. However, by setting a value to one of the LDPs such that, for instance,

$$
L_{q}\left(\eta_{0}^{2}\right)=0
$$

for some integer $q$ (but $L_{k}^{(1)}\left(\eta_{1}^{2}\right) \neq 0$ for all $k$ ), we obtain that, by writing $\left|\psi_{s}\right\rangle$ in the number state representation,

$$
\left|\psi_{s}\left(\eta_{0}\right)\right\rangle=\frac{1}{N_{0}} \sum_{n=0}^{q} C_{n}^{(0)}|n\rangle
$$

(the argument of $\psi_{s}$ denotes the condition we apply, i.e., in Eq (12), the condition is on $\left.\eta_{0}\right)$ where

$$
C_{n}^{(0)}=\left(-\frac{\Omega_{0} e^{-\eta_{0}^{2} / 2}}{\Omega_{1} e^{-\eta_{1}^{2} / 2}}\right)^{n}(n !)^{1 / 2} \prod_{m=0}^{n-1} \frac{L_{m}\left(\eta_{0}^{2}\right)}{L_{m}^{(1)}\left(\eta_{1}^{2}\right)}, \quad C_{0}^{(0)}=1
$$

and

$$
N_{0}^{2}=\sum_{n=0}^{q}\left|C_{n}^{(0)}\right|^{2}
$$


is the normalization constant.

If, instead of condition (11), we choose

$$
L_{p}^{(1)}\left(\eta_{1}^{2}\right)=0
$$

(but $L_{k}\left(\eta_{0}^{2}\right) \neq 0$ for all $k$ ) we obtain the wave function

$$
\left|\psi_{s}\left(\eta_{1}\right)\right\rangle=\frac{1}{N_{1}} \sum_{n=p+1}^{\infty} C_{n}^{(1)}|n\rangle
$$

where now,

$$
C_{n}^{(1)}=\left(-\frac{\Omega_{0} e^{-\eta_{0}^{2} / 2}}{\Omega_{1} e^{-\eta_{1}^{2} / 2}}\right)^{n-p-1} \sqrt{\frac{n !}{(p+1) !}} \prod_{m=p+1}^{n-1} \frac{L_{m}\left(\eta_{0}^{2}\right)}{L_{m}^{(1)}\left(\eta_{1}^{2}\right)}, \quad C_{p+1}^{(1)}=1,
$$

and

$$
N_{1}^{2}=\sum_{n=p+1}^{\infty}\left|C_{n}^{(1)}\right|^{2}
$$

Combining both conditions (11) and (15), one would obtain (for $q>p$ )

$$
\left|\psi_{s}\left(\eta_{0}, \eta_{1}\right)\right\rangle=\frac{1}{N_{01}} \sum_{n=p+1}^{q} C_{n}^{(1)}|n\rangle,
$$

with

$$
N_{01}^{2}=\sum_{n=p+1}^{q}\left|C_{n}^{(1)}\right|^{2}
$$

In this way, by setting the conditions (11), (15) or both, we can engineer states in the following three zones of the Hilbert space, (a) from $|0\rangle$ to $|q\rangle$, (b) from $|p+1\rangle$ to $|\infty\rangle$, or (c) from $|p+1\rangle$ to $|q\rangle$. In the later case, by setting $q=p+1$ generation of the number state $|q\rangle$ is achieved.

We should remark, that by selecting further apart sidebands one would obtain a different spacing in Eqs (12), (16) and (19). For instance, by choosing $j=2$ and $k=0$ one would obtain only even or odd number states in those equations (depending in this case on initial conditions and $W(s)$, the angular distribution of spontaneus emission). Also, it should be noticed that one can use the parameters $j=m+1$ and $k=m$ (with $m \neq 0$ ) (in the single 
number state spacing case) to extend the possibilities of choosing LDPs. LDPs of the order of one (or less) are needed [for conditions (11) and (15)] which can be achieved by varying the geometry of the lasers. For example, by setting $\eta_{0}=1$, we have $L_{1}\left(\eta_{0}^{2}=1\right)=0$, and therefore we obtain the qubit

$$
\left|\psi_{s}\left(\eta_{0}=1\right)\right\rangle=\frac{1}{\sqrt{1+\left|\frac{\Omega_{0}}{\Omega_{1}}\right|^{2} e^{\eta_{1}^{2}-1}}}\left(|0\rangle-\frac{\Omega_{0} e^{-1 / 2}}{\Omega_{1} e^{-\eta_{1}^{2} / 2}}|1\rangle\right),
$$

where by changing the Rabi frequencies, one has control of the amplitudes.

Finally, note that we could have also chosen to drive the $q$ th upper sideband instead of the $k$ th lower sideband in equation (21) with bassically the same results.

In conclusion, we have shown that specific superpositions of number states with $k$ th spacing emerge from the long time dynamics, when laser and ion decouple and this stops fluorescing. From Eq (19) one notes that we can generate robust to noise qubits with controllable amplitudes, which depend on the Rabi frequencies $(\Omega$ 's). This can be easily done by varying the LDPs to fulfil conditions (11) and (15). In this way, one has broad possibilities to "filter" (choose) which number states are present in the state to be generated.

H. M.-C. acknowledges W. Vogel and S. Wallentowitz for comments and M. Fortunato for discussions and proof-reading of the manuscript. H. M.-C. would also like to thank support from the Mexican Consejo Nacional de Ciencia y Tecnología (CONACYT). 


\section{REFERENCES}

[1] B. Sherman and G. Kurizki, Phys. Rev. A 45, R7674 (1992); B.M. Garraway, B. Sherman, H. Moya-Cessa, P.L. Knight, and G. Kurizki, Phys. Rev. A 49, 535 (1994); K. Vogel, V.M. Akulin, and W.P. Schleich, Phys. Rev. Lett. 71, 1816 (1993).

[2] G.M. D’Ariano, L. Maccone, M.G.A. Paris, and M.F. Sacchi, LANL e-print archive quant-ph/9906077.

[3] R.L. de Matos Filho and W. Vogel, Phys. Rev. Lett. 76, 608 (1996).

[4] R.L. de Matos Filho and W. Vogel, Phys. Rev. A 54, 4560 (1996).

[5] H. Moya-Cessa, S. Wallentowitz, and W. Vogel, Phys. Rev. A 59, 2920 (1999); see also: H. Moya-Cessa, S. Chávez-Cerda, and W. Vogel, J. Mod. Optics in press.

[6] M. Fortunato, G. Kurizki, and W.P. Schleich, Phys. Rev. A 59, 714 (1999).

[7] D. Vitali, P. Tombesi, and G.J. Milburn, Phys. Rev. Lett. 79, 2442 (1997); Phys. Rev. A 57, 4930 (1998).

[8] R.L. de Matos Filho and W. Vogel, Phys. Rev. A 58, R1661 (1998).

[9] A. Ekert and C. Macchiavello, Acta Phys. Pol. A 93, 63 (1998).

[10] S. Wallentowitz, W. Vogel and P.L. Knight, Phys. Rev. A 59, 531 (1999).

[11] H. Moya-Cessa, A. Vidiella-Barranco, J.A. Roversi, Dagoberto Freitas S., and S.M. Dutra, Phys. Rev. A 59, 2518 (1999). 